(C) 1996 IEEE. Personal use of this material is permitted. However, permission to reprint/republish this material

for advertising or promotional purposes or for creating new collective works for resale or redistribution to servers

or lists, or to reuse any copyrighted component of this work in other works must be obtained from the IEEE.

\title{
PHASE AND SYNCHRONOUS DETECTOR THEORY AS APPLIED TO BEAM POSITION AND INTENSITY MEASUREMENTS
}

\author{
J. D. Gilpatrick, Los Alamos National Laboratory, Los Alamos, NM 87545 USA
}

\begin{abstract}
A popular signal processing technique for beam position measurements uses the principle of amplitude-modulation -tophase-modulation (AM/PM) conversion and phase detection. This technique processes position-sensitive beam-imagecurrent probe-signals into output signals that are proportional to the beam's position. These same probe signals may be summed and processed in a different fashion to provide output signals that are proportional to the peak beam current. This paper derives the transfer functions for the AM/PM beam position and peak beam current processors.
\end{abstract}

\section{INTRODUCTION}

Presently, there are several techniques that process signals from position-sensitive beam-image-current probes into widebandwidth signals proportional to charged particle beam positions and peak current. One of the more popular techniques uses a double balanced mixer (DBM) as a component in a AM/PM beam-position processor and peak beam current or intensity detector $[1,2]$. The DBM is used as a phase detector in the beam position processor and a synchronous detector in the peak current processor. Fig. 1 and 5 show simplified schematics of theses two processors.

The processor initially filters and amplifies opposite probe-lobe signals (e.g., top and bottom lobes) to provide optimum measurement signal-to-noise characteristics. The amplitude ratio of these signals is then converted to a phase difference using an AM/PM circuit. The input signals to the $\mathrm{AM} / \mathrm{PM}$ circuit are

$\left|V_{T}\right| \sin \left(\omega_{R F} t+0\right)$

and

$$
\left|V_{B}\right| \sin \left(\omega_{R F} t+\pi / 2\right)
$$

where $\left|V_{T}\right|$ and $\left|V_{B}\right|$ are the amplitudes of a specific fundamental or harmonic component of the beam bunching frequency, $\omega_{R F}$. The $\pi / 2$ phase delay in the bottom lobe's signal is required for the correct operation of the particular AM/PM circuit realization shown in Fig. 1. The phase difference between the two AM/PM-circuit-output ports, $\Delta \theta$, is

$\Delta \theta=2 \arctan \left(10^{R / 20}\right)-\pi / 2$

where $R(d B)=20 \log \left(\left|V_{T}\right| /\left|V_{B}\right|\right)$ [2,3]. This AM/PM outputphase difference is measured by a DBM. Because of the inherent amplitude sensitivity of the DBM to amplitude variations, limiting amplifiers or limiters are used to stabilize the signal amplitudes to the DBM input ports in both the position and peak-current processors. These limiters are key components to an accurate AM/PM beam-position processor and must have minimal amplitude and phase delay variations throughout their dynamic range. A low pass filter (LPF) between the output amplifier and DBM removes all intermediate frequency (IF) signal components that are generated by limiter output-signals and DBM "mixing" process so that the processor's output signal is only proportional to the beam's position.

In the following analysis, only the DC transfer function will be considered. To add the transient behavior of these circuits, the temporal response of the final multi-pole lowpass-filter must be added to the position- and currentprocessor output equations.

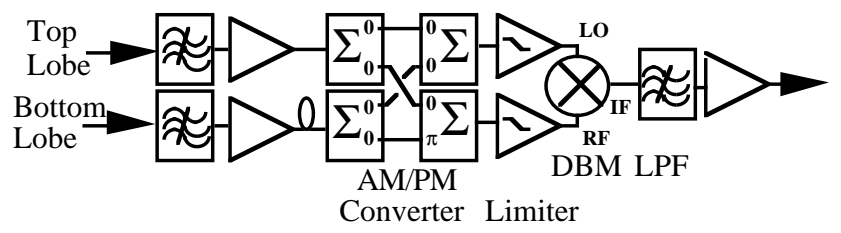

Figure 1: This schematic is a AM/PM beam-position processor. The DBM has two input ports commonly known as the local oscillator (LO) and radio frequency (RF) ports and an output IF port.

\section{GENERAL DBM OPERATION}

The DBM is a three port device consisting of two transformers whose primary and secondary windings are connected to two input ports and a bridge diode circuit, respectively (see Fig. 2). The DBM output port produces a

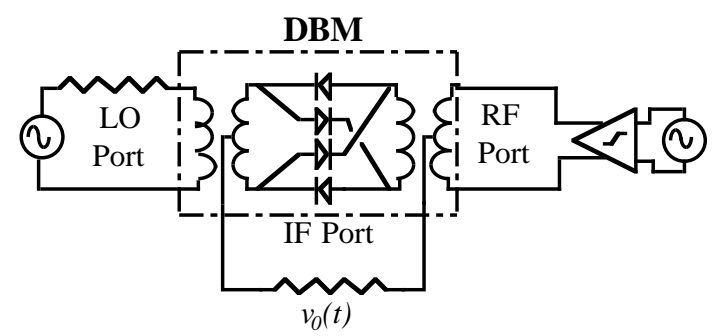

Figure 2: DBM schematic with a limiter amplifier driving the LO port and a terminating resistor across the output IF port.

current through a termination resistor connected between the center taps of the two secondary transformer-windings. As the sign changes on either the LO or RF input ports, currents are conducted through the appropriate diodes in the bridge circuit and through the termination resistor to produce an output voltage, $v_{0}(t)$.

The LO port is near saturation (as defined by the limiter drive circuitry and the DBM diodes in saturation), the resulting signal provides a square wave switching function described by 
$\mathrm{S}(\mathrm{t})=2 \sum_{\mathrm{n}=1}^{\infty} \frac{\sin (\mathrm{n} \pi / 2)}{\mathrm{n} \pi / 2} \cos \left(\mathrm{n} \omega_{L O} \mathrm{t}+\theta_{L O}\right)$.

If the RF port is not saturated, its signal is

$v_{R F}(t)=V_{R F} \cos \left(\omega_{R F} t+\theta_{R F}\right)$.

The resultant IF-output-port signal is the multiplication of these two input port signals [4]. Processing at a single frequency (i.e., let $\omega_{\mathrm{LO}}=\omega_{\mathrm{RF}}=\omega$ ) and folding the left frequency-domain components into the right components, therefore providing an additional factor of two, the unfiltered IF-output-port signal, $v_{0}(t)$, is

$$
\begin{aligned}
v_{0}(t)= & \frac{8}{\pi} V_{R F} \cos \left(\omega t+\theta_{R F}\right) \\
& \sum_{n=1}^{\infty} \frac{\sin (n \pi / 2)}{n} \cos \left(n \omega t+\theta_{L O}\right) .
\end{aligned}
$$

By employing a trigonometric identity, the two cosine terms with different arguments in Eq. (5) are separated into sum and difference frequency and phase terms and, $v_{0}(t)$ becomes

$$
\begin{gathered}
v_{0}(t)=\frac{8}{\pi} V_{R F} \sum_{n-1}^{\infty} \frac{\sin (n \pi / 2)}{n}\left\{\left[\operatorname { c o s } \left((\omega-n \omega) t+\left(\theta_{R F}-\right.\right.\right.\right. \\
\left.\left.\left.\left.\theta_{L O}\right)\right)\right]+\left[\cos \left((\omega+n \omega) t+\theta_{R F}+\theta_{L O}\right)\right]\right\} .
\end{gathered}
$$

Since only the fundamental components are used for the position and peak current processing, and remembering that the $\mathrm{LO}$ and RF ports have the same fundamental frequencies, the filtered IF-output-port signal, $v_{0}(t)$, is

$$
v_{0}(t)=\frac{4}{\pi} V_{R F} \sum_{n=1}^{\infty} \frac{\sin (n \pi / 2)}{n} \cos \left(\theta_{R F}-\theta_{L O}\right)(7)
$$

or

$$
v_{0}(t)=\frac{\beta}{\pi} V_{R F} \cos (\Delta \theta)
$$

where $\beta \approx 3.105$ for $n=1$ to 199 .

\section{POSITION TRANSFER FUNCTION}

\section{Unsaturated DBM RF-Port Case}

Fig. 1 shows the beam position processor with limiters connected to the RF- and LO-input DBM ports. To initially simplify the mathematical model, assume that there is no limiter connected to the DBM RF port (i.e., similar to Fig. 5). The position-processor transfer function, $v_{p o s}(t)$, is the combination of Eq. (2) and (8) and is

$$
v_{\text {pos }}(t)=\frac{\beta}{\pi} A_{I} A_{O} \alpha V_{R F} \cos \left[2 \arctan \left(10^{R / 20}\right)-\pi / 2\right]
$$

where $\alpha$ is an dimensionless attenuation circuit-constant and $A_{I}$ and $A_{O}$ are the input and output amplifier gains in volts per volts.

\section{Saturated DBM RF-Port Case}

However, for the typical realistic position processor, there are limiters for each DBM input port, therefore, Eq. (4) and (5) are rewritten to include the square-wave saturation effect of the limiters so that

$$
v_{R F}(t)=2 V_{R F} \sum_{m=1}^{\infty} \frac{\sin (m \pi / 2)}{m \pi / 2} \sin \left(m \omega_{R F} t+\theta_{R F}\right)
$$

and

$$
\begin{aligned}
v_{0}(t)= & 8 V_{R F} \sum_{n=1}^{\infty} \sum_{m=1}^{\infty} \frac{\sin (n \pi / 2)}{n \pi / 2} \frac{\sin (m \pi / 2)}{m \pi / 2} \\
& \cos \left(m \omega_{R F} t+\theta_{R F}\right) \cos \left(n \omega_{L O} t+\theta_{L O}\right) .
\end{aligned}
$$

The unfiltered DBM-IF-port signal is further simplified by recognizing that Eq. (11) is the convolution of two square waves and can be expressed as a Fourier series. After the same trigonometric identity used between Eq. (5) and (6) is applied, and higher frequency components are removed using a LPF, the filtered DBM-IF-port signal becomes

$v_{0}(t)=2 V_{R F}\left\{\frac{8}{\pi^{2}} \sum_{n=1}^{\infty} \frac{\sin \left[(2 n-1)\left(\theta_{L O}-\theta_{R F}\right)\right]}{(2 n-1)^{2}}\right\}$.

Fig. 3 shows the triangular waveform as a function of the DBM input-phase difference described by the bracketed portion of Eq. (12) [5].

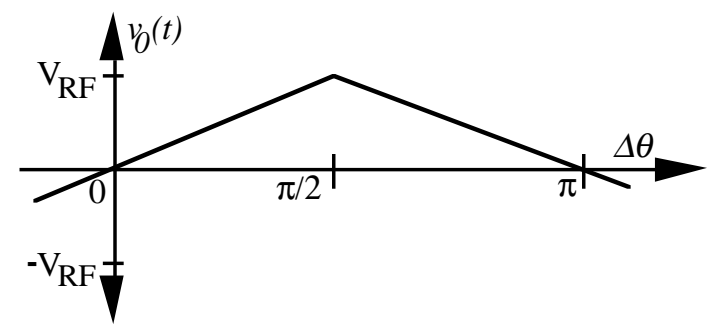

Figure 3: Waveform of $v_{0}(t)$ in Eq. (12).

Since the AM/PM converter does not allow the phase difference between the two DBM input ports to extend past $\pm \pi / 2$, Eq. (12) is further simplified as

$v_{0}(t)=\frac{\beta}{\pi} V_{R F}(\Delta \theta)$

where $\beta=4$. Therefore, for the saturated DBM-RF-port condition, the position-processor transfer-function equation is

$v_{\text {pos }}(t)=\frac{4}{\pi} A_{O} \alpha V_{R F}\left[\arctan \left(10^{R / 20}\right)-\pi / 4\right]$.

Fig. 4 shows, in practice, how well this model describes the actual circuitry. The circuit parameters $\alpha, A_{O}$, and $V_{R F}$ were measured for various input signal ratios, and a nonlinear least squares fit based on Eq. (14) was performed on the measured data. The fit variable was a single multiplier substituted for $\alpha, A_{O}$, and $V_{R F}$. As can be seen, the error between the fit and theory is within a $\pm 0.1 \mathrm{~dB}$ band. The measured centeredbeam multiplier for a $1 \mathrm{~dB}$ input ratio was 0.76 volts and the fitted equation gave a 0.79 volt multiplier. This difference is within the measurement errors of the circuit parameters used in the multiplier. 


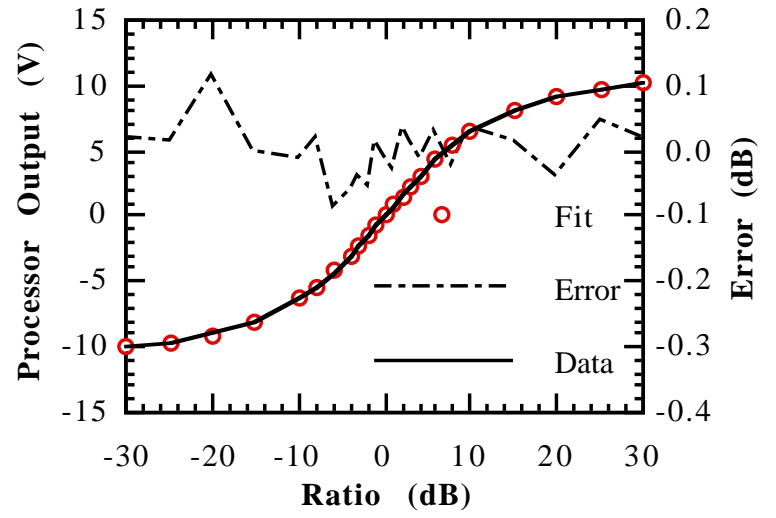

Figure 4: Measured data and fitted theory based on Eq. (14) and the error between the theory and data are plotted as a function of the ratio of opposite-lobe input signals.

\section{Alternate Form}

The position-processor transfer function can be further simplified by letting $\phi=\arctan \left(\left|V_{T}\right| /\left|V_{B}\right|\right)$ and using another trigonometric identity. The resultant simplification to Eq. (14) is

$$
v_{\text {pos }}(t)=\frac{4}{\pi} A_{O} \alpha V_{R F} \arctan \left[\frac{\tan \phi-1}{\tan \phi+1}\right] .
$$

Substituting for $\phi$ in Eq. (15), $v_{p o s}(t)$ is

$$
v_{\text {pos }}(t)=\frac{4}{\pi} A_{O} \alpha V_{R F} \arctan \left(\frac{\left|V_{T}\right|-\left|V_{B}\right|}{\left|V_{T}\right|+\left|V_{B}\right|}\right) .
$$

For beam positions near the center of the probe, Eq. (15) can be further reduced to

$$
v_{p o s}(t)=\frac{4}{\pi} A_{O} \alpha V_{R F}\left(\frac{\left|V_{T}\right|-\left|V_{B}\right|}{\left|V_{T}\right|+\left|V_{B}\right|}\right) .
$$

As can be seen from Eq. (16) and (17), the AM/PM ratio processing technique is a special case of the more generic "difference-over-sum" $(\Delta / \Sigma)$ processing technique. This $\mathrm{AM} / \mathrm{PM}$ technique has a larger dynamic range than other $\Delta / \Sigma$ processing techniques due to its inherent larger signal-to-noise characteristics for centered-beam conditions. Other typical $\Delta / \Sigma$ processors have lower signal-to-noise characteristics because most circuit realizations have an amplitude-nulling node or junction within their circuit topology. This nulling junction limits the generic $\Delta / \Sigma$-processor's dynamic-range for center beam conditions. As can be seen in Fig. 1, the AM/PM position processor does not have an amplitude-nulling junction within its circuit topology, and therefore, has a larger dynamic range than other $\Delta / \Sigma$ processors.

\section{CURRENT TRANSFER FUNCTION}

Fig. 5 shows how the DBM is used in a synchronous detector for the peak beam-current processor. There are two major differences between the beam position processor as shown in Fig. 1 and the peak beam current processor. First,

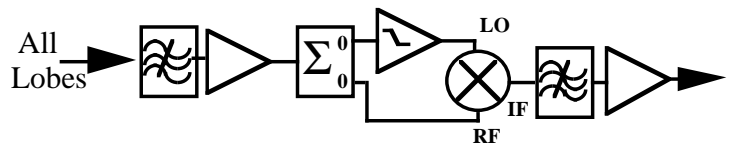

Figure 5: This schematic shows a peak current detector.

the peak current detector uses only a single limiter and, second, the LO and RF DBM ports have 0 or $\pi$ phase difference between them. This phase shift allows the RF port to act as a linear or synchronous detector for the signal presented to it (i.e., the DBM is a high-frequency full-wave rectifier for the RF-port sinusoidal-signal). The peak-currentprocessor transfer-function equation is Eq. (8) with $\Delta \theta$ equal to 0 or $\pi$, and $V_{R F}=A_{I} \alpha v_{L O B E S} / \sqrt{ } 2$ where $v_{\text {LOBES }}$ is the summation of all probe-lobe signals. The resultant transfer function, $v_{\text {current }}(t)$, is

$v_{\text {current }}(t)=\frac{\beta}{\sqrt{2} \pi} A_{I} A_{O} \alpha v_{\text {LOBES }}$

where, again, $\beta \approx 3.105$.

\section{CONCLUSION}

This paper has derived the transfer functions for the AM/PM beam position and peak current processors. The equations were solved for the nonsaturated and saturated DBM-RF-port beam-position-processor. The arctan function's argument, found in the typical saturated RF-port case, is comprised of the ratio of opposite probe lobes. After further simplification, it was found that this AM/PM positionprocessor circuit is really a special case of the more general "difference-over-sum" processing technique. Finally, the peak-beam-current transfer function was derived.

\section{REFERENCES}

[1] R. E. Shafer, "Beam Position Monitoring," AIP Conference Proceedings 212, Upton, NY, (1989).

[2] S. P. Jachim, R. C. Webber, and R. E. Shafer, "RF Beam Position Measurement for Fermilab Tevatron," IEEE Trans. Nucl. Sci. 28, 2323 (1981).

[3] J. D. Gilpatrick and F. D. Wells, "Double Balance Mixer Operation used as Phase and Synchronous Detectors as Applied to Beam Position and Intensity Measurements," LAUR-93-1622, AT-3 TECHNICAL NOTE:92-8, July, (1992).

[4] Solid__State _ Radio_ Engine Raab, John Wiley \& Sons Inc., 1980, p. 188-202.

[5] Continuous and Discrete Signal and Systems Analyssis, McGillen and Cooper, Holt Reinhart and Winston Inc., 1974, p. 101. 